\title{
Chain control sets and fiber bundles
}

\author{
CARLOS J. BRAGA BARROS* \\ Departamento de Matemática, Universidade Estadual de Maringá \\ 87020-900 Maringá, PR, Brasil \\ E-mail: cjbbarros@uem.br
}

\begin{abstract}
Let $S$ be a semigroup of homeomorphisms of a compact metric space $M$ and suppose that $\mathcal{F}$ is a family of subsets of $S$. This paper gives a characterization of the $\mathcal{F}$-chain control sets as intersection of control sets for the semigroups generated by the neighborhoods of the subsets in $\mathcal{F}$. We also study the behavior of $\mathcal{F}$-chain control sets on principal bundles and their associated bundles.
\end{abstract}

Mathematical subject classification: 93B03, 93B05.

Key words: semigroups, control sets, chain control sets, principal bundles, associated bundles.

\section{Introduction}

The concept of chain control set for control systems was introduced by Colonius and Kliemann [4], [7]. These sets appear as a tool for the analysis of the asymptotic properties of control systems (see [4], [5], [6], [7]). Extending this notion for general classes of semigroups Braga Barros and San Martin [2] defined chain control sets for a family of subsets of a semigroup acting on a homogeneous space. In that paper chain control sets were characterized as intersection of control sets for the semigroups generated by the neighborhoods of the subsets in the family. This paper shows a similar result for semigroups of homeomorphisms of a compact metric space. The approach is different from that of [2] because the semigroup may have empty interior in the group of homeomorphisms. An important fact which permits our generalization of [2] is that the hypothesis $\mathrm{H}$

\#460/98. Received: 30/X/98. Accepted: 11/XI/03.

*Research partially supported by CAPES/PROCAD - Teoria de Lie e Aplicações, grant n. 00186/00-7. 
defined there can be extended to semigroup of homeomorphisms acting on a compact metric space.

Apart from this general characterization of chain control sets we also study chain control sets on fiber bundles. The action of semigroups in fiber bundles arises naturally in many contexts. For instance in nonlinear control systems the linearized flow evolves on a fiber bundle over the state space of the system (see [4], [7]). The action of semigroups of diffeomorphisms on fiber bundles were studied by Braga Barros and San Martin [3]. In [3] the control sets were described from their projections onto the base space and their intersections with the fibers.

In this paper we pursue the same kind of results for chain control sets. We show that a chain control set in the total space of a fiber bundle projects inside a chain control set in the base space. On the other hand, we also show that a chain control set in the fiber is contained in a chain control set in the total space. Assuming that the structural group of a principal bundle is compact we use the characterization of chain control sets as intersections of control sets to show that the inverse image by the projection of a chain control set in the base space of the fiber bundle is a chain control set in the total space.

We believe that these topological results are useful for further developments of the dynamical aspects of control systems.

\section{$2 \mathcal{F}$-chain control sets}

In this section we recall the concept of a chain control set associated with a family of subsets of a semigroup. We refer to [2] for the definition of chain control sets for semigroup actions and corresponding results on homogeneous spaces of Lie groups.

For a semigroup of homeomorphisms of a compact metric space it is shown, under certain conditions, that a chain control set is the intersection of control sets for the semigroups generated by the neighborhoods of the subsets in the family.

We begin by assuming that $S$ is a semigroup of homeomorphisms of a compact metric space $M$. From now on, and in the whole paper we assume that $S$ and $S^{-1}$ have the accessibility property, that is, $\operatorname{int}(S x) \neq \emptyset$ and $\operatorname{int}\left(S^{-1} x\right) \neq \emptyset$ for every $x \in M$. 
We fix a distance $d$ on $M$ and define chain control sets for a family $\mathcal{F}$ of subsets of $S$.

Definition 1. Let $\mathcal{F}$ be a family of subsets of $S$. Take $x, y \in M$, a real $\varepsilon>0$ and $A \in \mathcal{F}$. A $(S, \varepsilon, A)$-chain from $x$ to $y$ consists of points $x_{0}=x, x_{1}, \ldots$, $x_{n-1}, x_{n}=y$ in $M$ and $\phi_{0}, \ldots, \phi_{n-1} \in A$ such that $d\left(\phi_{j}\left(x_{j}\right), x_{j+1}\right)<\varepsilon$ for $j=0, \ldots, n-1$. A subset $E \subset M$ is called a $\mathcal{F}$-chain control set if it satisfies

1. $\operatorname{int}(E) \neq \emptyset$.

2. For every $x, y \in E$, there exists a $(S, \varepsilon, A)$-chain from $x$ to $y$, for every $\varepsilon>0$ and $A \in \mathcal{F}$.

3. $E$ is maximal satisfying these properties.

It is easy to see that the notion of $\mathcal{F}$-chain control set does not change if an equivalent distance is considered. Also, it follows from the third condition that two $\mathcal{F}$-chain control sets are either disjoint or coincident. Furthermore, an application of Zorn's Lemma shows that any subset satisfying the first two conditions in the Definition 1 is contained in a $\mathcal{F}$-chain control set.

Next, we show that effective control sets (for the theory of control sets we refer to [11], [7]) are contained in $\mathcal{F}$-chain control sets. We need to impose the following conditions on $\mathcal{F}$.

Definition 2. Let $\mathcal{F}$ be a family of subsets of a semigroup $S$. We say that $\mathcal{F}$ satisfies property $P_{l}$ (respectively $P_{r}$ ) if for every $\phi, \psi \in S, \phi \neq 1$ and every $A \in \mathcal{F}$, there exists a positive integer $n$ such that $\phi^{n} \psi \in A$ (respectively $\left.\psi \phi^{n} \in A\right)$.

Let's denote by $S_{A}$ the semigroup generated by a subset $A \subset S$.

Proposition 1. Assume that $\mathcal{F}$ is a family of subsets of a semigroup $S$ satisfying the properties $P_{l}$ and $P_{r}$ and take $A \in \mathcal{F}$. Let $D$ be an effective control set for $S$. Then $D \subset \operatorname{cl}\left(S_{A} x\right)$ for any $x \in D$.

Proof. Take $x, y \in D$. Let us show that $y \in \operatorname{cl}\left(S_{A} x\right)$. Let $D_{0}=\{x \in D: x \in$ $\left.\operatorname{int}(S x) \cap \operatorname{int}\left(S^{-1} x\right)\right\}$ be the core of $D$. We know that $D_{0}$ is nonempty by the 
definition of effectiveness. Suppose first that $y \in D_{0}$. Since $D \subset \operatorname{int}\left(S^{-1} y\right)$ for every $y \in D_{0}$ (see [3], Proposition 2.2), there exists $\psi \in S$ such that $y=\psi(x)$. Also, there exists $\phi \in S-\{1\}$ such that $\phi(y)=y$. By $P_{l}$ we have $\phi^{n} \psi \in A$, and $\phi^{n} \psi(x)=y$, so that $y \in S_{A} x$. For an arbitrary $y$ take $z \in D_{0}$. We have seen that $z \in S_{A} x$. We have to show that $y \in \operatorname{cl}\left(S_{A} z\right)$. Take $\phi \in S-\{1\}$ such that $\phi(z)=z$ and a sequence $\psi_{m} \in S$ such that $\psi_{m}(z) \rightarrow y$. By $P_{l}, \psi_{m} \phi^{n} \in S_{A}$ for large enough $n$, and since $\psi_{m} \phi^{n}(z)=\psi_{m}(z)$, there exists a sequence $\rho_{k} \in S_{A}$ with $\rho_{k}(z) \rightarrow y$.

As a consequence we have.

Corollary 1. Suppose that $\mathcal{F}$ satisfies both $P_{l}$ and $P_{r}$. Then any effective control set for $S$ is contained in a $\mathcal{F}$-chain control set.

We can define effective $\mathcal{F}$-chain control sets.

Definition 3. Let $\mathcal{F}$ be a family of subsets of $S$. A $\mathcal{F}$-chain control set is called effective if it contains an effective control set for $S$.

We observe that for a family $\mathcal{F}$ satisfying both $P_{l}$ and $P_{r}$ a $\mathcal{F}$-chain control set $E$ is effective if and only if the subset $E_{0}=\left\{x \in E: x \in \operatorname{int}(S x) \cap \operatorname{int}\left(S^{-1} x\right)\right\}$ is not empty. In fact, if we assume that $x \in \operatorname{int}(S x) \cap \operatorname{int}\left(S^{-1} x\right)$ then there exists an effective control set $D$ such that $x \in D_{0}$ (see [3], Proposition 2.3).

Let $M$ be a compact metric space. Assume that $S$ is a semigroup of homeomorphisms of $M$ and suppose that $d$ is a metric on $M$. In the group $G$ of homeomorphisms of $M$ we consider the metric of uniform convergence

$$
d^{\prime}(\phi, \psi)=\sup _{x \in M} d(\phi(x), \psi(x))
$$

which is right invariant under $G$. For a subset $A \subset S$ we put

$$
B(A, \varepsilon)=\left\{\phi \in G: \exists \psi \in A, d^{\prime}(\phi, \psi)<\varepsilon\right\} .
$$

We denote by $S_{\varepsilon, A}$ the subsemigroup of $G$ generated by $B(A, \varepsilon)$.

We intend to show that points reachable by chains can be reached by the action of the perturbed semigroup $S_{\varepsilon, A}$. For this it is required to consider the following assumption about the action of $G$ on $M$. 
Hypothesis $\mathrm{H}$ : There exist real numbers $c>0$ and $\eta>0$ satisfying the following property: for all $x \in M$ and $y \in B_{\eta}(x)$, there exists $\phi \in G$ such that $\phi(x)=y$ and $d(\phi(x), x) \geq c d^{\prime}\left(\phi, 1_{M}\right)$.

In general this condition is not satisfied.

Example 1. Consider the compact metric space

$$
M=\bigcup_{n=1}^{\infty} C_{n} \cup\{0\}
$$

where $C_{n}$ are the circles $C_{n}=\left\{(x, y) \in \mathbb{R}^{2}: x^{2}+y^{2}=1 / n\right\}$, with the metric inherited from the standard metric of $\mathbb{R}^{2}$. All homeomorphisms $\phi$ of $M$ have the property that $\phi(0)=0$, and therefore the hypothesis $\mathrm{H}$ is not satisfied at 0 . In fact, if $\phi(0) \neq 0$ then the connected component containing $\phi(0)$ is a circle. The connected component containing 0 is the set $\{0\}$. And clearly this set in not homeomorphic to a circle.

However, we observe that in [2] it was shown that hypothesis $\mathrm{H}$ holds for the action of a Lie group $G$ on a large class of homogeneous spaces $G / H$, namely those for which there is a compact subgroup $K \subset G$ which acts transitively on $G / H$. This class of homogeneous spaces includes the flag manifolds (Furstenberg boundaries) of semi-simple Lie groups $G$.

Now, we can relate $(S, \varepsilon, A)$-chains with reachability of $S_{\varepsilon, A}$.

Proposition 2. Take $x, y \in M$ and $A \subset S$. Then

1. There exists an $(S, \varepsilon, A)$-chain from $x$ to $y$ if $y \in S_{\varepsilon, A} x$. We also have that there is a $\left(S, \varepsilon^{\prime}, A\right)$-chain from $x$ to $y$ for every $\varepsilon^{\prime}>\varepsilon$ if $y \in \operatorname{cl}\left(S_{\varepsilon, A} x\right)$.

2. Suppose that the hypothesis $H$ is satisfied and take $\varepsilon$ such that $0<\varepsilon<\eta$. Assume that $x_{0}, \ldots, x_{n} \in M$ and $\phi_{0}, \ldots, \phi_{n-1}$, determine $a(S, \varepsilon, A)$ chain from $x_{0}$ to $x_{n}$. Then there exists $\psi \in S_{\varepsilon^{\prime}, A}$ such that $\psi\left(x_{0}\right)=x_{n}$, where $\varepsilon^{\prime}=\varepsilon / c$.

Proof. 1. Take $y \in S_{\varepsilon, A} x$. Then, there exists $\phi \in S_{\varepsilon, A}$ such that $y=\phi(x)$. It follows from the definition of $S_{\varepsilon, A}$ that $\phi=\phi_{k-1} \ldots \phi_{0}$ with $\phi_{i} \in B(A, \varepsilon), i=$ $0, \ldots, k-1$. We choose $\psi_{0}, \ldots, \psi_{k-1} \in A$ with $d^{\prime}\left(\psi_{i}, \phi_{i}\right)<\varepsilon, i=0 . ., k-1$. 
The sequences $x_{0}, x_{1}=\phi_{0}\left(x_{0}\right), \ldots, x_{k}=\phi_{k-1}\left(x_{k-1}\right)=y$ and $\psi_{0}, \ldots, \psi_{k-1}$ determine an $(S, \varepsilon, A)$-chain from $x$ to $y$. In fact,

$$
\begin{aligned}
d\left(\psi_{i-1}\left(x_{i-1}\right), x_{i}\right) & =d\left(\psi_{i-1}\left(x_{i-1}\right), \phi_{i-1}\left(x_{i-1}\right)\right) \\
& \leq d^{\prime}\left(\psi_{i-1}, \phi_{i-1}\right)<\varepsilon
\end{aligned}
$$

for $i=0, \ldots, k-1$. Consequently there exists a $(S, \varepsilon, A)$-chain from $x$ to $y$. Now, suppose that $y \in \operatorname{cl}\left(S_{\varepsilon, A} x\right)$. There exists a sequence $\phi_{n} \in S_{\varepsilon, A}$ such that $\phi_{n}(x)$ converges to $y$. Take $\varepsilon^{\prime}>\varepsilon$ and let $n_{0}$ be such that $d\left(\phi_{n_{0}}(x), y\right)<$ $\varepsilon^{\prime}-\varepsilon$. As before, there exist a $(S, \varepsilon, A)$-chain from $x$ to $\phi_{n_{0}}(x)$. Let $y_{0}=$ $x, \ldots, y_{n}=\phi_{n_{0}}\left(x_{0}\right) \in M, \psi_{0}, \ldots, \psi_{n-1} \in A$ be a $(S, \varepsilon, A)$-chain from $x$ to $\phi_{n_{0}}(x)$. Thus $d\left(\psi_{i}\left(y_{i}\right), y_{i+1}\right)<\varepsilon$ for $i=0, \ldots, n-1$. Therefore, the chain $z_{0}=x, z_{1}=y_{1}, \ldots, z_{n-1}=y_{n-1}, z_{n}=y$ and $\psi_{0}, \ldots, \psi_{n-1} \in A$ determine an $(S, \varepsilon, A)$-chain from $x$ to $y$. In fact,

$$
\begin{aligned}
d\left(\psi_{n-1}\left(y_{n-1}\right), y\right) & \leq d\left(\psi_{n-1}\left(y_{n-1}\right), \phi_{n_{0}}(x)\right)+d\left(\phi_{n_{0}}(x), y\right) \\
& <\varepsilon^{\prime}
\end{aligned}
$$

and $d\left(\psi_{i-1}\left(y_{i-1}\right), y_{i}\right)<\varepsilon<\varepsilon^{\prime}$ for $i=1, \ldots, n-1$.

2. Since $d\left(\phi_{i}\left(x_{i}\right), x_{i+1}\right)<\varepsilon<\eta$, the hypothesis $\mathrm{H}$ implies that there is $\psi_{i} \in G$ such that

$$
d\left(\psi_{i}\left(\phi_{i}\left(x_{i}\right)\right), x_{i+1}\right)=d\left(\psi_{i}\left(\phi_{i}\left(x_{i}\right)\right), \phi_{i}\left(x_{i}\right)\right) \geq c d^{\prime}\left(\psi_{i}, 1_{M}\right)
$$

$i=0, \ldots, n-1$, and therefore $d^{\prime}\left(\psi_{i}, 1_{M}\right)<\varepsilon / c=\varepsilon^{\prime}$. Define $\tau_{i}=\psi_{i} \phi_{i}$. We have

$$
d^{\prime}\left(\tau_{i}, \phi_{i}\right)=d^{\prime}\left(\psi_{i} \phi_{i}, \phi_{i}\right)=d^{\prime}\left(\psi_{i}, 1_{M}\right)<\varepsilon^{\prime}
$$

because $d^{\prime}$ is right invariant. Therefore, $\tau_{i} \in B\left(A, \varepsilon^{\prime}\right)$. On the other hand, $\tau_{i}\left(x_{i}\right)=\psi_{i} \phi_{i}\left(x_{i}\right)=x_{i+1}$, and $x_{n}=\tau_{n-1} \ldots \tau_{0}\left(x_{0}\right)$.

Proposition 3. Suppose that the hypothesis $H$ is satisfied and assume that $\mathcal{F}$ satisfies $P_{l}$ and $P_{r}$. Let $D$ be an effective control set for $S$ on $M$. Then, for each $\varepsilon>0$ and $A \in \mathcal{F}$ there exists a control set $D_{\varepsilon, A}$ for the perturbed semigroup $S_{\varepsilon, A}$ such that $D \subset D_{\varepsilon, A}$. 
Proof. In fact, for $x, y \in D$, we will show that $y \in S_{\varepsilon, A} x$, for $\varepsilon$ small enough. By Proposition 1, there exists, $\phi \in S_{A}$ such that $\phi(x)$ is near $y$. On the other hand, by the definition of $S_{A}$ we have $\phi=\phi_{1} \ldots \phi_{n}$ with $\phi_{i} \in A$. Using $\mathrm{H}$, there is $\gamma$ with $d^{\prime}(\gamma \phi, \phi)=d^{\prime}(\gamma, 1)<\varepsilon$ and $\gamma \phi(x)=y$ so that $\gamma \phi \in S_{\varepsilon, A}$ and $y \in S_{\varepsilon, A} x$.

The next theorem characterizes the $\mathcal{F}$-chain control sets as intersections of control sets for the perturbed semigroups.

Theorem 1. Let $S$ be a semigroup of homeomorphisms of a compact metric space $M$. Suppose that the action of $S$ on $M$ satisfies $H$, and consider a family $\mathcal{F}$ of subsets of $S$ satisfying $P_{l}$ and $P_{r}$. Let $D$ be an effective control set for $S$ on $M$, and for $\varepsilon>0$ and $A \in \mathcal{F}$, denote by $D_{\varepsilon, A}$ the $S_{\varepsilon, A}$ control set containing $D$. Then

$$
E=\bigcap_{\varepsilon, A} D_{\varepsilon, A}
$$

is the only $\mathcal{F}$-chain control set containing $D$.

Proof. Since $D \subset E$ we have $\operatorname{int}(E) \neq \emptyset$. Also, for any $x, y \in E, y \in$ $\operatorname{cl}\left(S_{\varepsilon^{\prime}, A} x\right)$ for all $\varepsilon^{\prime}>0$ and $A \in \mathcal{F}$. Therefore, by the Proposition 2 there exists a $(S, \varepsilon, A)$-chain from $x$ to $y$ for all $\varepsilon>0$ and $A \in \mathcal{F}$, which shows that $E$ is chain transitive. It remains to verify the maximality of $E$. Take $x \notin E$ and $y \in E$ and suppose that for every $\varepsilon>0$ and $A \in \mathcal{F}$ there are $(S, \varepsilon, A)$-chains from $x$ to $y$ and from $y$ to $x$. Since the action of $S$ on $M$ satisfies $H$ and $S_{\varepsilon, A} \subset S_{\varepsilon^{\prime}, A}$ for $\varepsilon^{\prime}>\varepsilon$, Proposition 2 shows that $y \in S_{\varepsilon, A} x$ and $x \in S_{\varepsilon, A} y$ for all $\varepsilon>0$ and $A \in \mathcal{F}$. However, $y \in D_{\varepsilon, A}$, so that $x \in D_{\varepsilon, A}$ for all $\varepsilon, A$ contradicting the assumption that $x \notin E$. Hence, there is no chain either from $x$ to $y$ or from $y$ to $x$, which shows the maximality of $E$.

Proposition 4. Let the assumptions be as in the previous theorem and suppose moreover that there is just one invariant control set, say $D$, for $S$ in $M$. Then $D_{\varepsilon, A}$ is the $S_{\varepsilon, A}$-invariant control set containing the invariant control set for $S$. 
Proof. By [1, Lemma 3.1] there is only one closed invariant control set, say $C$, for $S$ in a compact metric space $M$ if and only if $C=\bigcap_{x \in M} \operatorname{cl}(S x) \neq \emptyset$. Define $C_{\varepsilon, A}=\bigcap_{x \in M} \operatorname{cl}\left(S_{\varepsilon, A} x\right)$. Thus, it is enough to show that $D_{\varepsilon, A} \cap C_{\varepsilon, A} \neq \emptyset$. Pick $x \in M$. Since $D \subset \operatorname{int}\left(S^{-1} y\right)$ for every $y \in D_{0}$ we can choose $\phi \in S$ such that $\phi(x) \in D_{0}$. But $\mathcal{F}$ satisfies property $P_{l}$ and therefore $\phi^{n} \in A$ for some integer $n$ (take $\phi=\psi$ in the definition of $P_{l}$ ). We have that $\phi^{n}(x) \in D$ because $D$ is invariant. Since $D \subset D_{\varepsilon, A}$, and $D_{\varepsilon, A}$ is a control set for $S_{\varepsilon, A}$ it follows that $D_{\varepsilon, A} \subset \operatorname{cl}\left(S_{\varepsilon, A} x\right)$.

\section{Fiber bundles}

In this section we study the behavior of $\mathcal{F}$-chain control sets on principal bundles and their associated bundles. We refer to [9] for the theory of fiber bundles.

We start by settling some notation. Let $G$ be a topological group. Suppose that $G$ acts effectively and on the right on a topological space $Q$. We denote by $Q(M, G)$ the principal bundle with total space $Q$, base space $M$ and structural group $G$. We denote by $\pi_{Q}: Q \rightarrow M$ the canonical projection.

Let $S_{Q}$ be a semigroup of homeomorphisms of $Q$ commuting with the right action, i.e., $Q \phi(q \cdot a)=Q \phi(q) \cdot a, a \in G$ if $Q \phi \in S_{Q}$. The semigroup $S_{Q}$ induces a semigroup $S_{M}$ of homeomorphisms of $M$. In fact, if $y \in M$ and $y=\pi_{Q}(q)$ we define an element $M \phi \in S_{M}$ as

$$
M \phi(y)=\pi_{Q}(Q \phi(q)),
$$

if $Q \phi \in S_{Q}$.

Suppose that the structural group $G$ acts on the left and transitively on the topological group $F$. We consider the fiber bundle associated to the principal bundle $Q(M, G)$ with typical fiber $F$. This bundle is denoted by $E(M, F, G, Q)$, or simply by $E$, the total space of the bundle. Since we will be interested in the study of chain control sets we assume, in the paper, that $E, Q$ and $M$ are metric spaces.

The elements of $E$ are equivalence classes with respect to the relation on $Q \times F$ given by $(q, v) \sim\left(q a, a^{-1} v\right), a \in G$. We use the notation $[q, v], q \in Q, v \in F$ for an element of $E$.

The canonical projection $\pi_{E}: E \rightarrow M$ on the fiber bundle is defined as $\pi_{E}([q, v])=\pi_{Q}(q)$. 
Let $Q \phi$ be a homeomorphism of $Q$ commuting with the right action of $G$. Then $Q \phi$ induces the homeomorphism of $E$ defined by $E \phi([q, v])=[Q \phi(q), v]$. Therefore the semigroup $S_{Q}$ of homeomorphisms of $Q$ induces a semigroup $S_{E}$ of homeomorphisms of $E$ and

$$
S_{E}([q, v])=\left[S_{Q}(q), v\right]
$$

Given $q \in Q$ we define the subset

$$
S_{q}=S_{Q}(q) \cap \pi_{Q}^{-1}(x), x=\pi_{Q}(q)
$$

Through the identification of the fiber over $x$ with $G$ via $a \in G \longmapsto q \cdot a \in$ $\pi_{Q}^{-1}(x), S_{q}$ can be viewed as a subset of $G$

$$
S_{q}=\left\{a \in G: \exists Q \phi \in S_{Q}, Q \phi(q)=q \cdot a\right\}
$$

It follows immediately that $S_{q}$ is a subsemigroup of $G$ if $S_{q} \neq \emptyset$. We observe that $S_{q}$ is the subsemigroup acting on the typical fiber.

The semigroups $S_{Q}, S_{E}$ and $S_{q}$ were also considered in [3].

Suppose that $\mathcal{F}$ is a family of subsets of $S_{Q}$. The family $\mathcal{F}$ induces a family $\mathcal{F}_{M}$ in the semigroup $S_{M}$, a family $\mathcal{F}_{E}$ in $S_{E}$ and a family $\mathcal{F}_{q}$ in $S_{q}$. In fact, for each $A \in \mathcal{F}$ we define $A_{M}=\left\{M \phi \in S_{M}: \exists Q \phi \in A\right.$ and $M \phi\left(\pi_{Q}(q)\right)=$ $\left.\pi_{Q}(Q \phi(q))\right\}, A_{E}=\left\{E \phi \in S_{E}: \exists Q \phi \in A\right.$ and $\left.E \phi([q, v])=[Q \phi(q), v]\right\}$ and $A_{q}=\{a \in G: \exists Q \phi \in A$ and $Q \phi(q)=q \cdot a\}$. Thus we can define the induced families $\mathcal{F}_{M}=\left\{A_{M}: A \in \mathcal{F}\right\}, \mathcal{F}_{E}=\left\{A_{E}: A \in \mathcal{F}\right\}$ and $\mathcal{F}_{q}=\left\{A_{q}: A \in \mathcal{F}\right\}$.

Proposition 5. Let $\mathcal{F}$ be a family of subsets of $S_{Q}$ satisfying both $P_{l}$ and $P_{r}$. Then $\mathcal{F}_{M}, \mathcal{F}_{E}$ and $\mathcal{F}_{q}$ also satisfy $P_{l}$ and $P_{r}$.

Proof. In fact, take $A \in \mathcal{F}$. There exists $n$ such that $Q \phi(Q \rho)^{n}=\sigma \in A$ if $Q \phi, Q \rho \in S_{Q}$. Thus

$$
M \sigma\left(\pi_{Q}(q)\right)=\pi_{Q}\left(\left(Q \phi(Q \rho)^{n}\right)(q)\right)=\left(M \phi(M \rho)^{n}\right)\left(\pi_{Q}(q)\right)
$$

and therefore $M \phi(M \rho)^{n} \in A_{M}$. For the family $\mathcal{F}_{E}$ we have

$$
E \phi(E \rho)^{n}([q, v])=E \phi\left(\left[(Q \rho)^{n}(q), v\right]\right)=\left[\left(Q \phi\left(Q \rho^{n}\right)\right)(q), v\right]
$$


and $E \phi(E \rho)^{n} \in A_{E}$. Now, take $a, b \in S_{q}$ and $A_{q} \in \mathcal{F}_{q}$. Then there exist $Q \phi, Q \psi \in S_{Q}$ such that $Q \phi(q)=q \cdot a$ and $Q \psi(q)=q \cdot b$. Thus $Q \phi(Q \psi)^{n}(q)=Q \phi\left(q \cdot a^{n}\right)=q \cdot b a^{n}$. Since $Q \phi(Q \psi)^{n} \in A$ we have $b a^{n} \in A_{q}$. We argue in the same way for the $P_{r}$ property.

The following theorem shows that chain control sets in the total space of a fiber bundle project into chain control sets in the base of the bundle.

Theorem 2. Let $E$ be a fiber bundle with projection $\pi: E \rightarrow M$. Let $\mathcal{F}$ be a family of subsets in $S_{Q}$. Suppose that $E$ is compact, and let $H \subset E$ be a $\mathcal{F}_{E}$-chain control set. Then there exists a $\mathcal{F}_{M}$-chain control set $B \subset M$ such that $\pi(H) \subset B$.

Proof. Since $\operatorname{int}(H) \neq \emptyset$ and $\pi$ is an open map, $\pi(H)$ has nonempty interior. Take $\varepsilon>0, A_{M} \in \mathcal{F}_{M}$ and $x^{\prime}, y^{\prime} \in \pi(H)$. Pick $x, y \in H$ such that $\pi(x)=x^{\prime}$ and $\pi(y)=y^{\prime}$. Let's show that there exists an $\left.\left(S_{M}, \varepsilon, A_{M}\right)\right)$ chain from $x^{\prime}$ to $y^{\prime}$. Since $E$ is compact, $\pi$ is uniformly continuous so that there is $\delta>0$ such that $d\left(\pi(z), \pi\left(z^{\prime}\right)\right)<\varepsilon$ if $d\left(z, z^{\prime}\right)<\delta, z, z^{\prime} \in E$. Let $x_{0}=x, x_{1}, \ldots, x_{n-1}, x_{n}=y$ in $E$ together with $E \phi_{0}, E \phi_{1}, \ldots, E \phi_{n-1}$ in $A_{E}$ form a $\left(S_{E}, \delta, A_{E}\right)$-chain from $x$ to $y$. Since $d\left(x_{j}, E \phi_{j}\left(x_{j}\right)\right)<\delta$ we have that $d\left(\pi\left(x_{j}\right), \pi\left(E \phi_{j}\left(x_{j}\right)\right)\right)=d\left(\pi\left(x_{j}\right), M \phi_{j}\left(\pi\left(x_{j}\right)\right)\right)<\varepsilon$ which shows that $\pi\left(x_{i}\right), M \phi_{i}$ determine a $\left.\left(S_{M}, \varepsilon, A_{M}\right)\right)$-chain from $x^{\prime}$ to $y^{\prime}$. Since $\pi(H)$ satisfies properties 1 and 2 in the definition of a $\mathcal{F}_{M}$-chain control set it is contained in a $\mathcal{F}_{M}$-chain control set.

We recall that we are assuming that $S_{Q}$ and $S_{Q}^{-1}$ have the accessibility property. However, since we wish to work over effective $\mathcal{F}_{M}$ - chain control sets, the following version of accessibility is needed.

Definition 4. Let $\mathcal{F}$ be a family of subsets of $S_{Q}$ satisfying both $P_{l}$ and $P_{r}$. Suppose that $H$ is an effective $\mathcal{F}_{M}$-chain control set in $M$ and take $H_{0}=\{x \in$ $\left.H: x \in \operatorname{int}\left(S_{M} x\right) \cap \operatorname{int}\left(S_{M}^{-1} x\right)\right\}$. The semigroup $S_{Q}$ is said to be accessible over $H_{0}$ if for every $q \in \pi_{Q}^{-1}\left(H_{0}\right), \operatorname{int}\left(S_{Q} q\right) \cap \pi_{Q}^{-1}\left(H_{0}\right) \neq \emptyset$.

Lemma 1. Let $H \subset M$ be an effective $\mathcal{F}_{M}$-chain control set and suppose that $S_{Q}$ is accessible over $H_{0}$. Then $\operatorname{int}\left(S_{q}\right) \neq \emptyset$ if $q \in \pi_{Q}^{-1}\left(H_{0}\right)$. 
Proof. Take $Q \phi \in S_{Q}$ such that $Q \phi(q) \in \operatorname{int}\left(S_{Q} q\right) \cap \pi_{Q}^{-1}\left(H_{0}\right)$. There exists an effective control set $D$ for $S_{M}$ such that $x=\pi_{Q}(q) \in D_{0}$. Thus $\pi_{Q}(Q \phi(q))=$ $M \phi\left(\pi_{Q}(q)\right) \in D_{0}$ and there exists $Q \psi \in S_{Q}$ such that $M \psi\left(\pi_{Q}(Q \phi(q))\right)=x$, so that $Q \psi Q \phi(q)$ belongs to the same fiber as $q$. Now, $Q \psi Q \phi(q) \in \operatorname{int}\left(S_{Q} q\right)$ hence if we let $a \in G$ be such that $Q \psi Q \phi(q)=q \cdot a$ then $a \in \operatorname{int}\left(S_{q}\right)$ showing the lemma.

As a converse of Theorem 2, we have

Theorem 3. Let $E$ be a fiber bundle with projection $\pi: E \rightarrow M$. Let $\mathcal{F}$ be a family of subsets in $S_{Q}$ satisfying both $P_{l}$ and $P_{r}$. Suppose that $E$ is compact, $B \subset M$ is an effective $\mathcal{F}_{M}$-chain control set and that $S_{Q}$ is accessible over $B_{0}$. Then there exists an effective $\mathcal{F}_{E}$-chain control set $H \subset E$ and such that $\pi(H) \subset B$.

Proof. Take $x \in B_{0}=\left\{x \in B: x \in \operatorname{int}\left(S_{M} x\right) \cap \operatorname{int}\left(S_{M}^{-1} x\right)\right\}$. Then $x$ belongs to an effective control set $C$ contained in $B$ ([3, Proposition 2.3]). We have shown in [3, Proposition 3.4] that there exists an effective control set $D \subset E$ for $S_{E}$ with $\pi(D) \subset C$. Corollary 1 implies that $D$ is contained in a $\mathcal{F}_{E}$-chain control set, say $H$. Using the fact that chain control sets do not overlap and the Theorem 2 there exists a $\mathcal{F}_{M}$-chain control set $B \subset M$ satisfying $\pi(E) \subset B$.

Let $d_{E}$ be a metric on the total space $E$ of the fiber bundle with typical fiber $F$. Let also $d_{F}$ be a right invariant metric on the fiber $F$. Consider a metric $d_{M}$ on the base space $M$ such that the canonical projection $\pi_{E}: E \rightarrow M$ is Lipschitizian, that is, there exists a constant $c>0$ such that for every $x, y \in E$ we have $d_{E}(x, y) \leq c d_{M}\left(\pi_{E}(x), \pi_{E}(y)\right)$.

The characterization of the chain control sets as intersection of control sets of the perturbed semigroup gives us the following result.

Theorem 4. Let $E$ be an fiber bundle associated to the principal bundle $Q$ whose structural group is compact. Suppose that $M$ and $E$ are compact and that $Q$ is connected. Assume that $\mathcal{F}$ is a family of subsets of $S_{Q}$ satisfying $P_{l}$ and $P_{r}$. We also assume that the actions of the groups of homeomophisms on $M$ and $E$ satisfy the hypothesis $H$. Let $B$ be an effective $\mathcal{F}_{M}$-chain control set on $M$ and suppose that $S_{Q}$ is accessible over $B_{0}$. Then $\pi_{E}^{-1}(B)$ is an effective $\mathcal{F}_{E}$-chain 
control set on $E$. Therefore there are as many effective $\mathcal{F}_{E}$-chain control sets in $E$ as $\mathcal{F}_{M}$-chain control sets in $M$.

Proof. We first show that $\left(\left(S_{M}\right)_{\varepsilon, A_{M}}\right)_{E} \subset\left(S_{E}\right)_{c \varepsilon, A_{E}}$, that is, $E \phi \in\left(S_{E}\right)_{c \varepsilon, A_{E}}$ if $M \phi \in\left(S_{M}\right)_{\varepsilon, A_{M}}$. It is enough to show that $E \phi \in B\left(A_{E}, c \varepsilon\right)$ if $M \phi \in B\left(A_{M}, \varepsilon\right)$. This is true because

$$
\begin{aligned}
d^{\prime}(E \gamma, E \phi) & =\sup _{[q, v] \in E} d_{E}(E \gamma([q, v]), E \phi([q, v])) \\
& =\sup _{[q, v] \in E} d_{E}([Q \gamma(q), v],[Q \phi(q), v]) \\
& \leq c \sup _{[q, v] \in E} d_{M}\left(\pi_{Q}(Q \gamma(q)), \pi_{Q}(Q \phi(q))\right) \\
& =c d^{\prime}(M \gamma, M \phi) .
\end{aligned}
$$

By the Theorem 1 we have $B=\bigcap_{\varepsilon, A_{M}} D_{\varepsilon, A_{M}}$ where $D_{\varepsilon, A_{M}}$ is a control set for $\left(S_{M}\right)_{\varepsilon, A_{M}}$. Thus $\pi^{-1}(B)=\bigcap_{\varepsilon, A_{M}} \pi^{-1}\left(D_{\varepsilon, A_{M}}\right)$. Now, [3, Proposition 3.7 and Proposition 3.9] implies that $\pi^{-1}\left(D_{\varepsilon, A_{M}}\right)$ is a control set for $\left(\left(S_{M}\right)_{\varepsilon, A_{M}}\right)_{E} \subset$ $\left(S_{E}\right)_{c \varepsilon, A_{E}}$ and therefore $\pi^{-1}(B)$ is a $\mathcal{F}_{E}$ chain control set in $E$, again by the characterization of chain control sets as intersections of control sets.

The next theorem shows that a $\mathcal{F}_{q}$-chain control set in a fiber of a bundle is contained in a $\mathcal{F}$-chain control set in the total space.

Theorem 5. Let $E$ be the bundle associated to the principal bundle $Q$ with projections $\pi_{E}$ and $\pi_{Q}$, respectively. Suppose that $\mathcal{F}$ is a family of subsets of $S_{Q}$. Take $q \in \pi_{Q}^{-1}(x), x \in M$. Assume that $H$ is a $\mathcal{F}_{q}$-chain control set on $F$. Then

1. Any $\mathcal{F}_{q}$-chain control set in $\pi_{Q}^{-1}(x)$ is contained in a $\mathcal{F}$-chain control set in $Q$.

2. $[q, H]$ is contained in a $\mathcal{F}_{E}$-chain control set in $E$.

\section{Proof.}

1. Let $H$ be a $\mathcal{F}_{q}$-chain control set in $\pi_{Q}^{-1}(x)$. Pick $z, z^{\prime} \in H$. Then for every $\varepsilon>0$ and $A_{q} \in \mathcal{F}_{q}$ there exists $x_{0}=z, x_{1}, \ldots>, x_{n-1}, x_{n}=z^{\prime}$ in $\pi_{Q}^{-1}(x)$ and $>a_{0}, a_{1}, \ldots, a_{n-1} \in A_{q}$ such that $>d\left(x_{j} a_{j}, x_{j+1}\right)<\varepsilon$ for $j=0, \ldots, n-1$. Let $>Q \phi_{j} \in A$ be defined as $Q \phi_{j}(q)=q a_{j}$. Then 
$>x_{0}, \ldots, x_{n}$ and $Q \phi_{j}, j=0, \ldots, n-1$ determine a $>\mathcal{F}$-chain from $z$ to $z^{\prime}$.

2. Take $[q, v]$ and $\left[q, v^{\prime}\right]$ in $[q, H]$. Since $H$ is a $\mathcal{F}_{q}$-chain control set, for every $\varepsilon>0$ and $A_{q} \in \mathcal{F}_{q}$ there exist $v_{0}=v, \ldots, v_{n}=v^{\prime}$ in $F$ and $a_{0}, \ldots, a_{n-1} \in A_{q}$ such that $d_{F}\left(a_{j} v_{j}, v_{j+1}\right)<\varepsilon$ for $j=0, \ldots, n-1$. Let $Q \phi_{j} \in A$ be defined as $Q \phi_{j}(q)=q a_{j}$. Then $\left[q, v_{0}\right], \ldots,\left[q, v_{n}\right]$ and $E \phi_{j}, j=0, \ldots, n-1$ determine a $\left(S_{E}, \varepsilon, A\right)$-chain from $[q, v]$ to $\left[q, v^{\prime}\right]$. In fact,

$$
\begin{aligned}
d_{E}\left(E \phi_{j}\left(\left[q, v_{j}\right]\right),\left[q, v_{j+1}\right]\right) & =d_{E}\left(\left[q a_{j}, v_{j}\right],\left[q, v_{j+1}\right]\right) \\
& \left.=d_{E}\left(\left[q, a_{j} v_{j}\right]\right),\left[q, v_{j+1}\right]\right) \\
& =d_{F}\left(a_{j} v_{j}, v_{j+1}\right) \\
& <\varepsilon .
\end{aligned}
$$

Remark. Since we can construct the invariant control sets in the total space of an associated bundle in terms of the invariant control sets in the fibers (see [3]) we could ask if this is also true for the chain control sets which contains the invariant control set. We have tried to answer this question using the characterization of chain control sets as intersections of control sets (Theorem 1). The proof does not work because the semigroup associated (in the fiber) of the perturbed semigroup in the total space does not coincide with the perturbed semigroup of the fiber and there is no useful inclusion between them. We observe that Theorem 5 can be regarded as a lemma in this expected description of the chain control sets in fiber bundles.

\section{Acknowledgment}

I would like to thank an anonymous referee for suggestions to improve the paper, in particular for providing Example 1.

\section{REFERENCES}

[1] L. Arnold, W. Kliemann and E. Oeljeklaus, Lyapunov, A exponents of linear stochastic systems in: Lyapunov Exponents, Arnold L. and Wihstutz V. (eds), LNM-Springer 1186 (1986), Berlin. 
[2] C.J. Braga Barros and L.A.B. San Martin, Chain control sets for semigroup actions. Computational and Applied Mathematics, 15, 3 (1996), Birkhäuser, 257-276.

[3] C.J. Braga Barros and L.A.B. San Martin, On the action of semigroups in fiber bundles. Matemática Contemporânea, 13 (1997), Sociedade Brasileira de Matemática, 1-19.

[4] F. Colonius and W. Kliemann, Linear control semigroups acting on projective spaces. Journal of Dynamics and Differential Equations, 5, 3 (1993), 495-528.

[5] F. Colonius and W. Kliemann, Some aspects of control systems as dynamical systems. Journal of Dynamics and Differential Equations, 5, 3 (1993), 469-494.

[6] F. Colonius and W. Kliemann, Remarks on ergodic theory of stochastic flows and control flows. In Proceedings of the Conference on Stochastic Flows (Wihstutz V. ed.) Birkhäuser, Boston (1990).

[7] F. Colonius and W. Kliemann, The dynamics of control. Birkhäuser, Boston (2000).

[8] F. Colonius, W. Kliemann and L.A.B. San Martin, Asymptotic problems - from control systems to semigroups. Positivity in Lie Theory: Open Problems Walter de Gruyter GmbH \& Co. Berlin - New York (1998).

[9] D. Husemoller, Fibre Bundles. Graduate texts in Mathematics 20. Springer Verlag, New York Heidelberg Berlin (1975).

[10] S. Kobayashi and K. Nomizu, Foundations of differential geometry. John Wiley \& Sons, New York (1963).

[11] L.A.B. San Martin and P.A. Tonelli, Semigroup actions on homogeneous spaces. Semigroup Forum, 50 (1995), 59-88.

[12] L.A.B. San Martin, Control sets and semigroups in semi-simple Lie groups. In Semigroups in algebra, geometry and analysis. Gruyter Verlag, Berlin (1994). 